\title{
COGNITIVE METAPHORS OF THE MIND IN THE CANTERBURY TALES
}

\author{
AGNIESZKA WAWRZYNIAK
}

Adam Mickiewicz University Kalisz

agnieszka78kaga@wp.pl

\begin{abstract}
The paper presents an analysis of a number of cognitive metaphors pertaining to the concept of mind (e.g. sanity and insanity), heart, and fire. The study has been based on the text of Canterbury Tales by Geoffrey Chaucer. The paper contains a short theoretical introduction and a discussion of different linguistic and psychological approaches to issues related to figurative and literal, conventional language use. The analytical part focuses on the detailed contextual study of the cognitive metaphorical concepts. It is argued that many apparently similar concepts can evoke semantically conflicting metaphors, while concepts that appear to be mutually exclusive can sometimes evoke common associations and thereby similar metaphors.
\end{abstract}

Keywords: metaphor, metonymy, associations, culture-specific, universal

\section{Introduction}

The aim of the present paper is to analyse varieties of figurative expressions within the concept of the mind in The Canterbury Tales. The paper explores varieties of contexts from Middle English and analyses them in the light of contemporary theories of metaphors and metonymies. The theoretical part deals with contemporary theories on metaphor and metonymy continuum represented by Barcelona (2000), Radden (2000), Cruse (2004) among others, and with the theory of objectification (Szwedek 2007). The aim of the analytical part is to focus on the detailed contextual study of the metaphors linked with the concept of the mind and to view it in close relation with the theories on metaphor and metonymy continuum. Moreover, the analysis will show which metaphors in the corpus are culture-specific and which are more universal.

The analysis is based on all texts of Caxton's edition of The Canterbury Tales: The British Library Copies (ed. by Barbara Bordalejo), available as a CD-Rom containing the first full-colour facsimiles of all copies of William Caxton's first and second editions of Geoffrey Chaucer's The Canterbury Tales. It is also the first-ever electronic publication of the full text of the Caxton editions. 


\section{Theoretical prerequisites}

Following the traditional approach, metaphor involves mapping of more tangible, concrete phenomena (a source domain) upon abstract ones (a target domain) across different conceptual domains, while metonymy involves mapping of a source onto a target within one and the same conceptual domain. The traditional approach implies that metaphor and metonymy involve two distinct processes, the first one being based on similarity and the other on congruity. From that perspective, one can put a relatively clear-cut line between the metaphorical and metonymic processes.

It should be emphasised that the overall approach to the concepts of metaphor/ metonymy and their coreferentiality has changed with the publications of such linguists as Traugott (1989), Barcelona (2000), Radden (2000) and Cruse (2004). For instance, Radden, in his approach to metaphor and metonymy suggests that they cannot be conceived as discrete entities:

\footnotetext{
The traditional distinction between metaphor and metonymy can no longer be maintained. The classical notions of metaphor and metonymy are to be seen as prototypical categories at the end points of a continuum of mapping processes. The range in the middle of the metonymy-metaphor continuum is made up of metonymy-based metaphors, which also account for the transition of metonymy to metaphor by providing an experiential motivation of a metaphor (Radden 2000: 105).
}

One of the crucial points the newer cognitive approach highlights is that the distinction between metaphor and metonymy is scalar rather than discrete (cf. also Barcelona 2000:16). According to this new perspective, not only metaphor and metonymy need not be viewed as two opposite processes, but metaphor can be motivated metonymically thereby giving rise to metaphor based on metonymy. In other words, metonymy is a conceptual prerequisite for metaphor. Barcelona (2000) further illustrates this assumption claiming that motivation is not necessarily tied up with a sequential ordering of the mapping and that the mapping may even be simultaneous. Namely, the metonymic understanding of the source or target domain in a metaphor need not have become conventionalised prior to the conventionalisation of the metaphor. One example of metonymy-based metaphor, discussed by Barcelona, is the metonymic motivation of most metaphors for emotions (anger, happiness, sadness, love, and pride) on the basis of physiological or behavioural responses to emotions. For instance, a physiological effect of emotion that is often metonymised is the Affected Heart Rate, (the heart rate, which is believed to change as a result of a strong emotional impact stands for emotion), as in his heart rate stopped when he saw her. In other words, metaphor does not really develop 'out of' the metonymy but is motivated and constrained by the metonymic model of the target. Such an approach casts a different light upon both metaphor and metonymy and the relation between them. This new perspective on metaphor-metonymy continuum is applied in the present paper in the analysis of The Canterbury Tales expressions linked with the concept of the mind.

With regard to the theory of objectification, Szwedek (2007) emphasises that all nonphysical phenomena have to be conceptualised as physical objects before they can be assigned structure and orientation. By having no shape on their own, they are treated as objects and inherit all the properties of an object. Szwedek explains that objectification is 
the basis for all conceptualisation, as it is only the physical world that is directly accessible to our senses. The paper will attempt to show that the metaphor of objectification is a basis for the conceptualisation of wisdom and that different types of objects are involved in the conceptualisation of wisdom.

\section{The analysis of the concept of the mind}

The analysis of the concept of the mind requires an examination of various collocations from the conceptual point of view. The aim of the present section is to provide a threefold division within the concept of the mind and analyse the expressions that refer to the following components: high intellectual capacity, low intellectual capacity and madness. Moreover, the study will show that wisdom was highly objectified, which makes these metaphors more universal rather than culture-specific.

\subsection{High intellectual capacity}

The metaphors linked to the high intellectual capacity can be noticed in the following phrases:
(1) ful of sapience 'full of wisdom'
(2) haue sapience 'have wisdom'
(3) gret light of sapience 'great light of wisdom'
(4) fulfilled of sapience 'filled with wisdom'
(5) thoughtful 'thoughtful'
(6) high prudence 'high prudence'
(7) my wit is greet 'my wit is great'
(8) ouercome be reson 'overcome by reason'
(9) assay greet sapience 'test great wisdom'
(10) need of sapience 'need of wisdom'
(11) wit yeue 'give wit'

\subsubsection{Wisdom and the container metaphor}

Wisdom can be conceptualised as contents within a container, which can be observed is phrases $(1,4,5$, and 6 above $)$. In these contexts, the whole self functions as a container for thoughts, thus creating the metaphor THE SELF IS A CONTAINER FOR THOUGHTS. The amount of the substance in the container is indicative of the person's intelligence and erudition. In other words, the container has to be full for if the person is to be considered wise and perceptive. Moreover, contexts of phrases $(1,4,5$, and 6$)$ are also conceptualised along orientational metaphors MORE IS UP and REASON IS UP (Lakoff and Johnson 1980) where the more thoughts are placed in the container, the wiser the person is.

In the analysis of the container metaphor, two elements full and high can also be compared. In both, the container serves as an element enabling visualisation of wisdom. 
Metaphorically speaking, one can say that by putting many thoughts into the container, they will reach a higher level inside the container. Nevertheless, full marks two entities, the container as well as the content inside it. It gives information that there is a large amount of content, which is, however, externally bounded. The adjective high, however, is devoid of this information. High refers, first of all, to the container and describes its physical appearance. Only later this adjective is mapped on the more abstract level, where it indicates the level of wisdom. Thus high, from the purely physical description of the container, proceeds to stand for the description of the content itself, thereby giving rise to metonymy.

The context of (7) is also linked with the container metaphor, yet in this example, the wit corresponds to the container. Thus, the wit is conceptualised as great when it houses a large amount of thoughts. The analysis of the contexts, illustrated so far, shows that two types of containers are linked with wisdom, namely the self is a container for thoughts, as well as the wit is a container for thoughts. The conceptualisation of the self as the container for wisdom makes the instances metaphorical, while the projection of the wit as the container gives rise to metaphors based on metonymy, where a part stands for the whole. In other words, the part (here: great wit) stands for the whole person perceived as wise and erudite.

\subsubsection{Wisdom and the concept of light}

Wisdom can also be conceptualised via the concept of light, which can be exemplified by context (3). The example corresponds to the metaphor linked with light - IDEAS ARE LIGHT SOURCES (Lakoff and Johnson 1980). It seems that the two metaphors connected with wisdom, the container and the light metaphors, can be juxtaposed and differentiated. The container metaphor highlights the effect of absorbing knowledge, thus the variety of thoughts in the container. The intelligent and wise person is the one that knows a lot on many topics; the person that has collected multiplicity of information. In other words, the container metaphor focuses on what the person already knows; what he or she has in his/her mind. However, the light metaphor does not highlight the effect of gathering knowledge, but rather concentrates on the process of obtaining knowledge. Light makes us see. It penetrates everywhere and enlightens every dark and murky region casting a different perspective on it. An intelligent person is the one that does not see only one perspective or one dimension but a variety of aspects related to the topic. It is the person that is eager to penetrate through unknown areas and discuss difficult topics casting a different light or perspective on them. The light metaphor highlights the aspect of penetration, seeing many dimensions, and unveiling the mysterious, the unknown or taboo topics thereby structuring the process of gaining knowledge. One can even proceed further and claim that the expression greet light of sapience illustrates wisdom as a kind of light. Wisdom, hence, can be perceived as equipped with metaphorical light that emanates outward. Approaching these examples, light is a kind of instrument that enables to draw the value of wisdom. In other words, the source domain of light is used to conceptualise wisdom as a value in itself. Equipped with such attributes as uniqueness, goodness and eminence, the domain of light raises the value of wisdom, which becomes conceived as unique, eminent and ennobling. In 
other words, the element of light makes the person be perceived not only as wise, but also as noble. The two elements, namely wisdom and nobility, are blended in the WISDOM IS LIGHT metaphor. What takes place is the process of the transferring the attributes of light upon the target domain of wisdom which becomes linked with light, evokes highly positive undertones. Although the metaphorical correlation between wisdom and light is widely accepted in present-day English, wisdom itself seems to receive different connotations when compared with data from the corpus. In other words, PDE expressions such as "bright person" or "clear ideas" are neutral with respect to morality. Hence, they only refer to intelligence and do not imply any other undertones. In The Canterbury Tales, however, wisdom, similar to light, was evidently viewed as ennobling, unique and eminent. In other words, it contained the element of nobility in its conceptual framework.

\subsubsection{Wisdom as a commodity}

Middle English (ME) nouns denoting knowledge could co-occur in collocation with other verbs, such as have, govern, trust, overcome, test and prove, which can be seen in contexts $(2,8,9,10$, and 11$)$. Within the commodity metaphor, wisdom can be viewed as a valuable object, a weapon or a product.

In (2), wisdom is conceptualised as an object. A person has wisdom just like he or she could have any commodity, yet this commodity is perceived as something valuable. The context can bring to mind the metaphor WISDOM IS A VALUABLE OBJECT. The same metaphor is recorded in context (11), in which wit can be given. Here, wit can be viewed as a container, which is like a treasure that can change one's life completely.

In (8), wisdom is conceptualised via the metaphor REASON IS WEAPON, which can be seen in the following context:

And your wyf restrayne that wicked purpose

And ouercome you be reson and by good counceyll (The Tale of Melibee 124).

(And your wife will restrain that wicked purpose

And will overcome you by reason and by a good council).

Reason, then, is highly valued. The person, by thinking logically, can overcome the others. In (9) and (10), wisdom is conceptualised as a product:

Be cause of thy swete wordes and eke for I have

Assaied thy grete sapience and thy grete trouthe

I will gouern me by thy council in alle manner thing (The Tale of Melibee 146).

(Due to your sweet words and, moreover, for I have tested your great wisdom and your

great truth I will be directed by your council in all kinds of things).

If ony of yow have nede of sapience (The Tale of Malibee 151)

(If any of you have need of sapience).

In these contexts, wisdom can be tested just like any other product, or it may be felt to be needed. 


\subsection{Low intellectual capacity}

The metaphors linked to low intellectual capacity can be represented by the following expressions:

(12) rude wit 'rude wit'

(13) thynne wit 'thin wit'

(14) ful blynd conceit 'full blind conceit'

(15) wit suffices 'wit is sufficient'

(16) where was thy wit? 'where is your wit'

(17) short wit 'short wit'

\subsubsection{Low intellectual capacity preceded by adjectives centrally referring to a person}

In (12) and (14) wit is conceptualised as a person. Hence, they are personified or viewed as animate objects. In these contexts the adjectives rude and blind require some analysis as they have a different meaning when related to mind than when referring to a person. Hence, rude wit does not correspond to a rude person, whereas blind conceit does not stand for a blind person. These adjectives have been further metaphorised. Rude person denotes someone who speaks or behaves in a way that is not polite or is likely to offend or annoy people, while the expression rude wit is used to imply that the person is uneducated and ignorant. Nevertheless, the common platform of references can be found for the two collocations. Both expressions refer to some common norms which, however, have been violated. In the former phrase (rude person), the person does not abide by the generally accepted canon of behaviour, while in the latter phrase (rude wit), the person does not conform to the generally accepted canon of knowledge. Both manners are perceived as annoying. Furthermore, in the two expressions, the person chooses this way of conduct purposefully. In other words, he or she deliberately does not submit to the imposed rules existing in the society, either by rejecting received norms of behaviour, or by a denial of the generally accepted canon of knowledge. Additionally, in both expressions rude has evaluating, pejorative undertones.

A similar analysis can be carried out in case of blind. Blind in the expression blind person denotes someone who is physically handicapped and cannot see. Blind in blind conceit implies a person that is unenlightened and ignorant. Yet, as in the formerly analysed expression, a common platform of references can be found. To begin with, the person who has seen a lot, knows a lot. The process of knowing is thereby linked with seeing. Moreover, a wise person, just like light, is willing to penetrate everything and to mentally embrace as much as possible, which is within his or her field of vision. He or she is characterised by curiosity and inquisitiveness. Thus, the expression blind conceit implies that the person is unwilling to see. Being unwilling may entail the hypothesis that the person will not see and thus will remain blind and unaware of everything that is happening around. This perseverance not to see can be associated with indifference and lack of interest. The modification of blind with full further reinforces the passive attitude to knowledge. Hence, while the collocation blind person is linked with being physically handicapped, the phrase blind conceit is connected with being mentally handicapped. 
Blind person is thus a literal statement as the adjective blind encodes the basic, root sense. Nevertheless, blind conceit is a metaphorical expression in which blind denotes the epistemic, subjective sense. The expressions related to wit are also instances of metaphors based on metonymies (part for the whole), yet the adjectives should be also additionally perceived as equipped with metaphorical undertones.

\subsubsection{Low intellectual ability preceded by adjectives centrally referring to humans and objects}

The lexemes that pertained to mind, could also be modified by the adjectives that centrally referred both to humans and objects (e.g., thin wit, short wit). As for thin, the following central senses can be distinguished. When referred to objects, thin denotes a very small distance between two sides or two flat surfaces. Thin can also be linked with a person to point to someone that has little fat on his or her body. In both, the concept of a small amount is imprinted. A thin book is the one that contains few pages, while a thin person is someone that is slim hence there is little fat on his or her body. As for thin wit, it can be conceptualised as a long passage in which there are just few thoughts. Thus the adjective thin affects a different conceptualisation of wit. The image of wit that appears in the mind is not the one of a container but rather of a passage due to the modifying adjective. In the analysed expressions, this passage is projected as containing hardly any thoughts. Furthermore, the adjective thin, by evoking the mental content of the mind, is metaphorical as opposed to thin in "thin book" or "thin person", where it refers to the physical appearance of the described entities.

It should be emphasised, however, that thin, in the collocation with wit seems to be metaphorical to a lesser extent than blind and rude in the collocations with conceit and wit respectively. Blind and rude when used in collocation with the mind require a different meaning, or at least a highly motivated interpretation of the meaning that centrally refers to the physical appearance of a person. The semantics of thin collocating with book, person, or wit is roughly similar. It just needs to be slightly modified when applied to wit. In other words, the images of the book, person, or wit, when preceded by thin are similar in the sense that these entities appear to be of a certain length, yet they contain a small amount or number of something, namely of pages (book), fat (person) or thoughts (wit). As already noted, the number of pages or the amount of fat refers to the physical description of a book or a person, respectively. However, the number of thoughts is linked with the mental content of the wit, which, in turn, is connected with the metaphorisation of the adjective thin.

Wit could also be modified by the adjective short. The similar line of analysis can be observed both in short as in thin when juxtaposed with the wit. Short, like thin, occurs in collocations with the person as well as the object. Short, when referred to objects, denotes a small amount in length, e.g., as in "short hair." Linked with a person, short pertains to someone who is of less than average height. Short wit can be conceptualised as a short passage in which there are few thoughts due to the limitations of the passage. In other words, the passage is so short that it can hold only few thoughts. The juxtaposition of thin wit and short wit results in totally different images. In thin wit the passage is long but it contains very few thoughts. Additionally, these thoughts may 
appear as weak or feeble as they are housed in the thin passage. In short wit, the passage is so small that it physically cannot hold many thoughts. Furthermore, the adjective short in the collocation short wit is similarly metaphorical as thin in thin wit. Thus, the meaning of short in short hair, short person and short wit is similar, yet in the first two instances short refers to the physical description of the entities, while in the latter instance short applies to the mental analysis of the appearance and the content of the wit.

It should also be emphasised that the collocations linked with a low level of intelligence evoke the image of the passage, as opposed to the collocations that pertain to a high level of intelligence, which create the image of the container. One should emphasise that the attribute of the size is associated with the container image, which in turn is conducive to the large number of thoughts that could be housed inside it. On the other hand, the passage is also a kind of a container. Yet, by being marked by adjectives linked to the size or breadth, it becomes conceptualised as a container that is narrow or short. Such metaphors are based on metonymies in which the part stands for the whole.

\subsubsection{Wit as an enity temporarily absent}

Wit could also be conceptualised as an entity that is temporarily absent or exists in small amounts. In (16) wit is visualised as the entity temporarily absent, which can be noticed in the following context:

Where was thy wit? (The Maniciple's Tale 178).

(Where was your wit?).

Wit is viewed here as an entity that was once present but then it disappeared, thereby contributing to a chaos.

Wit, in (15) is conceived of as an entity present in small amounts, which can be seen in the following lines:

Now wolde my wit wolde suffise

To tellyn al that longith to that art. (The Canon's Yeoman's Prologue 162-163).

(Now I would like my wit would be sufficient enough to tell all that is appropriate to that art).

In this context the person is wondering if the possessed brain is big enough to be able to express all he wants to express. As in selected examples above, the analysed metaphors are based on metonymies, where the part stands for the whole.

\subsection{Madness}

The metaphors linked to madness can be noticed in the following phrases:

(18) out of mynde ('out of mind')

(19) half out of mynde ('half out of mind')

(20) lese hed ('lose head')

(21) lese wit ('lose wit') 
(22) mynde is goon ('mind is gone')

(23) troubil wit ('troubled wit')

(24) see woodness ('see madness')

(25) mad wit ('mad wit')

\subsubsection{Madness conceptualised as a totally or half absent mind}

Madness can be conceptualised as a totally or half absent mind. Mind, as totally absent is recorded in contexts (18) and (22), whereas it is half-absent in (19). Mind is commonly perceived as that part of humans that is responsible for rational and logical thinking. Therefore madness is frequently visualised as either total absence of, generally speaking, mind or as mind which exists only in certain fragments. In (18), the person is out of his mind. Consequently, mind is outside this person and he or she is separated from his or her mind. This context emphasises separation of the mind and the body. Yet this sentence does not invalidate the existence of the mind. It only implies that there is no connection between the mind and the body. The body does not receive the signals from the mind. A similar separation can be noticed in the collocation mynde is goon ("mind is gone"). Yet, additionally there is an implication that the mind disappeared somewhere. Thus, while in (18) (- out of his mynde-), such lack of connection is highlighted, in (22) (-mynde is goon-), both the separation from the body and the subsequent loss are highlighted.

Moreover, mind can also be viewed as a fragmentary structure. The person may appear to be partially separated from his or her mind. In other words, it is only a half or some part of the mind that seems to be outside of the human being. Therefore while in (18) (- out of his mynde-), no connection exists between the mind and the body, in (19), (- half out of mynde- ), the partial connection is maintained.

Another possible conceptualisation of madness is via metaphor based on metonymy, which can be exemplified by collocations (20) and (21), viz. lese hed and lese wit. The metonymy involved here is part for the whole, where the part (- hed and wit -), i.e. "head" and "wit" stand for the intellectual side of the human being. Additionally, hed and wit are conceptualised as lost objects, thereby giving rise to a metaphor MADNESS IS A LOST MIND/WIT/ HEAD. Consequently, mind, wit and hed are objectified. These examples are, thus, instances of metaphors that have a metonymic basis. They are dependent on the conceptually prior metonymic conceptualisation. In this model, one part, namely MIND/ HED or WIT is singled out to refer to the mental state of a person. The projection of a complete MIND/HED or WIT creates the image of a sane person. On the other hand, absent or half- absent mind gives rise to a person perceived as mad. There is thus a strong connection between a lost MIND/HED or WIT and a "gained" madness. Consequently, madness can be conceptualised as an absent or half-absent MIND/HED or WIT, as it is the direct consequence of MIND/HED or WIT having been lost. Madness in (21) is perceived as an outcome of a lost wit, which can be exemplified by the following context:

Ye fare lik a man hadde lost his wit (The Wife of Bath's Tale 1068).

(You behave like a man who has lost his wit). 
In other words, wit is responsible for human rational thinking. Yet, possessing wit may not be permanent as one can lose wit.

\subsubsection{Madness conceptualised as a perceivable object}

As for the conceptualisation of madness via objects, one should also notice that madness can also be visualised as a perceivable object. In other words, madness becomes an entity that can be seen. Nevertheless, in (24) it is not madness that one sees but rather reactions, ways of behaviour that show that someone is mad:

Yet saw I woodnesse in his rage (The Knight's Tale 1159).

(Yet I saw madness in his rage).

\subsubsection{Madness conceptualised as a troublemaker}

Furthermore, wit in (23) could also be viewed as a troublemaker, thereby evoking metaphor based on metonymy MADNESS IS A TROUBLE MAKER, which belongs to the category of animate objects. This metaphor can be exemplified by the context:

O troubil wit o ire reckless (The Maniciple's Tale 175).

(O troublesome wit, o reckless ire).

Hence, madness could be conceptualised via metaphors based on metonymies. The analysis shows that the collocations containing the element of wit, mynde and woodness are used with reference to madness.

Within the collocations centralising wit, the following metaphors based on metonymies can be distinguished: MADNESS IS AN ABSENT OR A HALF ABSENT MIND (lese wit), MADNESS IS A TROUBLEMAKER (troubil wit) and madness is an insane person (mad wit). In the metaphor MADNESS IS AN ABSENT OR A HALF ABSENT MIND, the "wit", viewed as a rational and logical tool is lost. Thus the wit possessed by the person was well-functioning but, unfortunately, the person is no longer its owner. In the metaphor MADNESS IS A TROUBLEMAKER, wit is viewed as possessing some failure. Moreover, wit is here visualised as a separate being that leads people astray.

As for the collocations including mynde, they also gave rise to metaphors based on metonymies due to the fact that mynde also stood for intellectual human side. The figurative expressions that contained mynde centralised both total and half-absence of the mind. Consequently, the connection between the mind and the body was either lost or imperfect. Within the general metaphors that project the total absence of the mind, two sub-models can be distinguished. One of them highlights only some separation of the mind from the body, but not the loss of the mind (out of mynde). The other one emphasises disappearance of the mind (mynde is goon). All these expressions view mynde as a logical, rational entity with which, however, the bond was severed.

With regard to collocations involving woodness, they give rise to pure metaphors. Woodness was the term used for mental illness and not a part of a human being. It gave 
rise to the metaphor MADNESS IS A PERCEIVABLE OBJECT. Following this metaphor, madness is then conceptualised as an entity that can be seen.

\section{Conclusions}

To conclude, the analysis of metaphors of the mind in Chaucer's The Canterbury Tales, as viewed in present day's perspective, led to the following conclusions:

To begin with, metaphors of the mind can be divided into universal and culturespecific. The universal metaphors are the ones which reflect traces of objectification. The metaphors which belong to this category are the following ones:

1. WISDOM IS CONTENTS IN A CONTAINER (contents is a kind of a substance, thus an object)

2. WISDOM IS A COMMODITY

3. WISDOM IS PRECEDED BY ADJECTIVES THAT CENTRALLY REFER TO HUMANS AND/OR OBJECTS (personified entities are animate objects)

4. WIT IS AN ENTITY TEMPORARILY ABSENT

5. MADNESS IS ABSENT OR HALF ABSENT MIND (the mind, similar to objects, can disappear)

6. MADNESS IS A PERCEIVABLE OBJECT

7. MADNESS IS A TROUBLEMAKER (animate object)

With regard to culture-specific metaphors, the conceptualisation of wisdom via light implies different undertones than in present-day English. When projected by light, wisdom contained not only elements of morality and nobility, but was also conceived of as unique and eminent. Moreover, the analysis also explored the structure of metaphors pertaining to a high level of intelligence, low level of intelligence and madness.

Within the collocations pertaining to a high level of intelligence, several metaphorical images can be distinguished. First of all, wisdom can be conceptualised as contents within the container. It should be emphasised that two types of the container are applied in the visualisation of wisdom, namely the self and the wit. The conceptualisation of the self as a container for wisdom makes the instances metaphorical, while the projection of the wit as a container gives rise to metaphors based on metonymies. In such contexts, wit (the part) stands for the whole intellectual side of the human being.

Moreover, wisdom can also be conceptualised as linked with light, thereby giving rise to a metaphor IDEAS ARE LIGHT SOURCES (Lakoff and Johnson 1980). The light metaphor highlights the effect of acquiring knowledge, not its effects.

Furthermore, wisdom is also viewed as a commodity. As for the conceptualisation of wisdom as the commodity, one can distinguish between metaphors that show wisdom as a valuable object and those which illustrate it as weapon.

As for the collocations centralising a low level of intelligence, the following metaphors can be distinguished. To begin with, wit can be personified. In such contexts, it is modulated by adjectives that centrally refer to humans. Yet, such adjectives refer to negative qualities of a person (e.g. rude) or his or her bodily afflictions (e.g. blind). 
Moreover, wit is also conceptualised as a passage either thin or short. A thin passage is both thin and contains few thoughts. The short passage is so small that it houses hardly any thoughts. In other words, the collocations that are linked with low intelligence evoke the image of a passage, as opposed to the collocations that pertain to a high level of intelligence, which create the image of the container.

Furthermore, low intelligence is linked with the metaphor IDEAS ARE LIGHT SOURCES (Lakoff and Johnson 1980). In such collocations the adjective blind is used to denote feeble-mindedness and a lack of intelligence.

In addition, the collocations connected with low intelligence project wit as an entity that is temporarily absent or exists in small portions. Such metaphors are contingent on the conceptually prior metonymy, where the part stands for the whole.

As for madness, the analysis has shown that it could also be conceptualised via varieties of metaphors based on metonymies. The collocations that refer to madness often contain such lexemes as wit, mynde and madness. Madness appears to be visualised metaphorically as the consequence of the loss of rational, logical tool (wit) or in terms of partial or whole separation of the mind from the body.

\section{References}

Barcelona, Antonio (2000). "On the possibility of claiming a metaphoric motivation for a conceptual metaphor" In A. Barcelona (ed.), Metaphor and metonymy at the crossroads. A cognitive perspective (31-58). Berlin: Mouton de Gruyter.

Bordelajo, Barbara (ed.) (2003). Caxton's Canterbury Tales Project: The British Library Copies. Birmingham: University of Birmingham.

Cruse, David Alan (2004). Meaning in language. An introduction to semantics and pragmatics. Oxford: Oxford University Press.

Lakoff George \& Mark Johnson (1980). Metaphors we live by. Chicago: University of Chicago Press.

Langacker, Ronald (1990). Concept, image and symbol: the cognitive basis of grammar. Berlin: Mouton de Gruyter.

Radden, Gunter (2000). "How metonymic are metaphors." In: A. Barcelona (ed.), Metaphor and metonymy at the cross-roads. A cognitive perspective (92-108). Berlin: Mouton de Gruyter.

Sweetser, Eve (1990). From etymology to pragmatics. Metaphorical and cultural aspects of semantic structure. Cambridge: Cambridge University Press.

Szwedek, Aleksander (2007). "Alternative theory of metaphorisation. In M. Fabiszak (ed.), Language and meaning (313-327). Frankfurt am Main: Peter Lang.

Traugott, Elisabeth (1989). "On the rise of epistemic meaning in English: an example of subjectification in semantic change." Language, 65, 31-55. 